\title{
The hedgehog pathway in breast cancer
}

\author{
Sheikh Asim Ali \\ Department of Medical Oncolgy, Temple University Hospital, Philadelphia, Pennsylvania, USA \\ Corresponding to: Sheikh Asim Ali, M.D, Associate Professor of Medicine. Temple University Medical School, Medical Oncologist/Hematologist, St \\ Luke's Health System, 701 Ostrum Street, Suite 403, Bethlehem, Pa 18015, USA. Email: Asim.Ali@sluhn.org.
}

Submitted Sep 25, 2012. Accepted for publication Oct 29, 2012.

DOI: $10.3978 /$ j.issn.1000-9604.2012.10.02

Scan to your mobile device or view this article at: http://www.thecjcr.org/article/view/1176/1509

Breast cancer is one of the most common cancers around the world with approximately 1.6 million new cases and 425 thousand deaths from the disease in 2010 (1). While early detection methods and treatment have continuously evolved and improved over the years, there is still a strong need to further understand the pathways and mechanisms that lead to and drive this malignancy with the ultimate goal being more personalized, less toxic treatment with improved survival and higher cure rates.

Inappropriate activation of the Hedgehog $(\mathrm{HH})$ pathway has been implicated in the development of cancers of the skin, brain, digestive tract, lung and prostrate (2-6). Aberrant reactivation of $\mathrm{HH}$ signaling has also been reported in breast cancer $(7,8)$. HH signaling is also thought to contribute to invasiveness $(9,10)$. GLi1 is thought to be a marker of $\mathrm{HH}$ pathway activation (11-13). It is part of the final step in the pathway which is regulated by the zinc finger transcription factors GLi1 and GLi2 which are activators of the $\mathrm{HH}$ target genes and GLi3 which acts as a repressor $(14,15)$. This pathway has been studied as a potential target in breast cancer (16). It has been demonstrated that a high expression of GLi1 by immunohistochemistry was an independent risk factor for skip metastasis of axillary lymph nodes in breast cancer (17). There has also been some data suggesting the targeting of this pathway in estrogen receptor negative breast cancer may be of benefit (18). A more recent article actually implicated this pathway in tamoxifen resistance and suggested it as a potential target in tamoxifen resistant breast cancer (19). This study demonstrated that a high GLi1 expression correlated inversely with disease free and overall survival in a cohort of 315 patients with breast cancer.

In this issue of the CJCR Zhang et al. report that the over expression of GLi1 in interstitial tissue from samples taken from breast cancer cases can serve as a prognostic factor for relapse and can be a marker for early relapse in this disease. Tissue from 284 cases of breast cancer seen at their centre between 2002 and 2004 was stained for GLi1 via IHC and the results were scored as high or low per a standardized Immuno-Reactive score (IRS). They demonstrated that tumor tissue had a higher level of Gli 1 expression than adjacent tissue and strongly correlated with HER2 status and age. The study also found a correlation between a high nuclear expression of GLi1 and an unfavorable recurrence free survival $(\mathrm{P}<0.05)$.

In light of the data that has accumulated so far and the data that is emerging including this important study by Zhang et al., activation of the hedgehog pathway signified by increased expression of GLi1 does seem to have a correlation with both the development of disease and its aggressiveness which makes this pathway a potential target for future therapy. A drug that inhibits this pathway has shown great promise in basal cell skin cancer (20) and multiple studies are underway with drugs that target this pathway in a multitude of malignancies including breast cancer. In addition to identifying patients who may benefit from targeting this pathway, Zhang et al.'s article demonstrates that markers like GLi1 could also serve as useful prognostic indicators to help better asses the risk of spread and aggressiveness of disease.

\section{Acknowledgements}

Disclosure: The author declares no conflict of interest

\section{References}

1. Forouzanfar MH, Foreman KJ, Delossantos AM, et al. Breast and cervical cancer in 187 countries between 1980 
and 2010: a systematic analysis. Lancet 2011;378:1461-84.

2. Beachy PA, Karhadkar SS, Berman DM. Tissue repair and stem cell renewal in carcinogenesis. Nature 2004;432:324-31.

3. Rubin LL, de Sauvage FJ. Targeting the Hedgehog pathway in cancer. Nat Rev Drug Discov 2006;5:1026-33.

4. Watkins DN, Berman DM, Burkholder SG, et al. Hedgehog signalling within airway epithelial progenitors and in small-cell lung cancer. Nature 2003;422:313-7.

5. Karhadkar SS, Bova GS, Abdallah N, et al. Hedgehog signalling in prostate regeneration, neoplasia and metastasis. Nature 2004;431:707-12.

6. El-Zaatari M, Tobias A, Grabowska AM, et al. Deregulation of the sonic hedgehog pathway in the InsGas mouse model of gastric carcinogenesis. Br J Cancer 2007;96:1855-61.

7. Cui W, Wang LH, Wen YY, et al. Expression and regulation mechanisms of Sonic Hedgehog in breast cancer. Cancer Sci 2010;101:927-33.

8. Souzaki M, Kubo M, Kai M, et al. Hedgehog signaling pathway mediates the progression of non-invasive breast cancer to invasive breast cancer. Cancer Sci 2011;102:373-81.

9. Feldmann G, Dhara S, Fendrich V, et al. Blockade of hedgehog signaling inhibits pancreatic cancer invasion and metastases: a new paradigm for combination therapy in solid cancers. Cancer Res 2007;67:2187-96.

10. Nagai S, Nakamura M, Yanai K, et al. Glil contributes to the invasiveness of pancreatic cancer through matrix metalloproteinase-9 activation. Cancer Sci 2008;99:1377-84.

11. Ruel L, Rodriguez R, Gallet A, et al. Stability and association of Smoothened, Costal2 and Fused with Cubitus interruptus are regulated by Hedgehog. Nat Cell

Cite this article as: Ali SA. The hedgehog pathway in breast cancer. Chin J Cancer Res 2012;24(4):261-262. DOI: 10.3978/ j.issn.1000-9604.2012.10.02
Biol 2003;5:907-13.

12. Lewis MT. Hedgehog signaling in mouse mammary gland development and neoplasia. J Mammary Gland Biol Neoplasia 2001;6:53-66.

13. Lewis MT, Ross S, Strickland PA, et al. Defects in mouse mammary gland development caused by conditional haploinsufficiency of Patched-1. Development 1999;126:5181-93.

14. Bai CB, Stephen D, Joyner AL. All mouse ventral spinal cord patterning by hedgehog is Gli dependent and involves an activator function of Gli3. Dev Cell 2004;6:103-15.

15. Kasper M, Regl G, Frischauf AM, et al. GLI transcription factors: mediators of oncogenic Hedgehog signalling. Eur J Cancer 2006;42:437-45.

16. Kubo M, Nakamura M, Tasaki A, et al. Hedgehog signaling pathway is a new therapeutic target for patients with breast cancer. Cancer Res 2004;64:6071-4.

17. Wang H, Mao XY, Zhao TT, et al. Study on the skip metastasis of axillary lymph nodes in breast cancer and their relation with Gli1 expression. Tumour Biol 2012. [Epub ahead of print].

18. Kameda C, Tanaka H, Yamasaki A, et al. The Hedgehog pathway is a possible therapeutic target for patients with estrogen receptor-negative breast cancer. Anticancer Res 2009;29:871-9.

19. Ramaswamy B, Lu Y, Teng KY, et al. Hedgehog Signaling Is a Novel Therapeutic Target in Tamoxifen-Resistant Breast Cancer Aberrantly Activated by PI3K/AKT Pathway. Cancer Res 2012;72:5048-59.

20. Sekulic A, Migden MR, Oro AE, Dirix L, et al. Efficacy and safety of vismodegib in advanced basal-cell carcinoma. N Engl J Med 2012;366:2171-9. 